\title{
The CALIPSO Mission and Initial Observations of Aerosols and Clouds from CALIOP
}

\author{
David M. Winker \\ NASA Langley Research Center , MS/475, Hampton, VA 23681 \\ david.m.winker@nasa.gov
}

\begin{abstract}
Launched in April 2006, the CALIPSO satellite provides unique global measurements of aerosols and clouds using a two-wavelength polarization lidar. This paper discusses mission status, instrument performance, and initial results.
\end{abstract}

OCIS codes: (280.1100) Aerosol detection; (280.3640) Lidar

\section{Introduction}

The CALIPSO satellite was launched on 28 April, 2006 and is now flying as part of the Afternoon Constellation (Atrain). CALIPSO was designed to provide unique measurements to improve our understanding of the role of aerosols and clouds in the Earth's climate system [1]. The CALIPSO satellite carries the Cloud Aerosol LIdar with Orthogonal Polarization (CALIOP, pronounced as "calliope"), the first polarization lidar in space, along with infrared and visible passive imagers.

CALIOP is a three-channel backscatter lidar optimized for aerosol and cloud profiling. Linearly polarized laser pulses are transmitted at $532 \mathrm{~nm}$ and $1064 \mathrm{~nm}$. The $1064 \mathrm{~nm}$ receiver channel is polarization insensitive, while the two $532 \mathrm{~nm}$ channels separately measure the components of the $532 \mathrm{~nm}$ backscatter signal polarized parallel and perpendicular to the outgoing beam. Profiles from CALIOP provide information on the vertical distributions of aerosols and clouds, cloud ice/water phase (via the ratio of signals in two orthogonal polarization channels), and a qualitative classification of aerosol size (via the wavelength dependence of the backscatter).

The current A-train configuration consists of five satellites, all flying at an altitude of $705 \mathrm{~km}$ in a sunsynchronous polar orbit, with an equator crossing time of about 1:30 PM local solar time. CALIOP is a nadirviewing instrument so it produces a curtain of profile data along the subsatellite point, providing sparse global coverage. The orbit inclination of $98^{\circ}$ provides coverage between $82^{\circ} \mathrm{N}$ and $82^{\circ} \mathrm{S}$. This paper presents a summary of the on-orbit performance of CALIOP and an overview of initial results.

\section{The CALIOP instrument}

CALIOP consists of a laser transmitter subsystem and a receiver subsystem. The transmitter subsystem includes two identical, redundant laser transmitters, each with a beam expander, and a beam steering system that ensures alinement between the transmitter and receiver. The Nd:YAG lasers produce simultaneous pulses at $1064 \mathrm{~nm}$ and $532 \mathrm{~nm}$ at a pulse repetition rate of $20.16 \mathrm{~Hz}$. Each laser generates $220 \mathrm{~mJ}$ of energy at $1064 \mathrm{~nm}$, which is frequency-doubled to produce $110 \mathrm{~mJ}$ of energy at each of the two wavelengths. A 1-meter telescope receives the backscattered light. Photomultiplier tubes (PMTs) are used to detect the $532 \mathrm{~nm}$ signal as they provide very low dark noise and reasonable quantum efficiency. The sensitivity and large linear dynamic range of the PMTs allows measurement of molecular scattering in the stratosphere as well as signals from dense boundary layer clouds. An avalanche photodiode (APD) is used at $1064 \mathrm{~nm}$, as PMT detectors have poor quantum efficiency at that wavelength. The APD has good dynamic range and quantum efficiency but the dark noise is much larger than for the PMTs. Thus the $532 \mathrm{~nm}$ channels are more sensitive. A rotating mechanism allows a depolarizer to be moved into the $532 \mathrm{~nm}$ beam for depolarization calibration. Key payload specifications are summarized in Table 1.

The backscattered signals are sampled at $10 \mathrm{MHz}$, corresponding to a 15 meter range increment. To match the bandwidth of the receiver electronics, the $532 \mathrm{~nm}$ channels are averaged to 30-m resolution and the $1064 \mathrm{~nm}$ channel is averaged to $60-\mathrm{m}$ resolution. To reduce requirements on downlink telemetry bandwidth, the lidar profile data is further averaged above an altitude of $8 \mathrm{~km}$, prior to downlinking.

Further details on the CALIOP instrument are given in [2]. Details on CALIOP retrieval algorithms and data products are given in [3], and can also be found on the CALIPSO web site: www-calipso.larc.nasa.gov. 
Table 1. Key characteristics of CALIOP

\begin{tabular}{|ll|}
\hline Laser & Nd:YAG \\
Pulse Energy & $110 \mathrm{~mJ}: 532 \mathrm{~nm}$ \\
Repetition Rate & $110 \mathrm{~mJ}: 1064 \mathrm{~nm}$ \\
Pulse Length & $20.16 \mathrm{~Hz}$ \\
Polarization Purity & $20 \mathrm{nsec}$ \\
Receiver FOV & $>1000: 1(532 \mathrm{~nm})$ \\
Vertical sampling & $130 \mu \mathrm{rad}$ \\
Footprint spacing & $10 \mathrm{MHz}(15 \mathrm{~m})$ \\
Linear Dynamic Range & $333 \mathrm{~m}$ \\
$\quad$ (all three channels) & $4 \mathrm{E}+6: 1$ \\
\hline
\end{tabular}
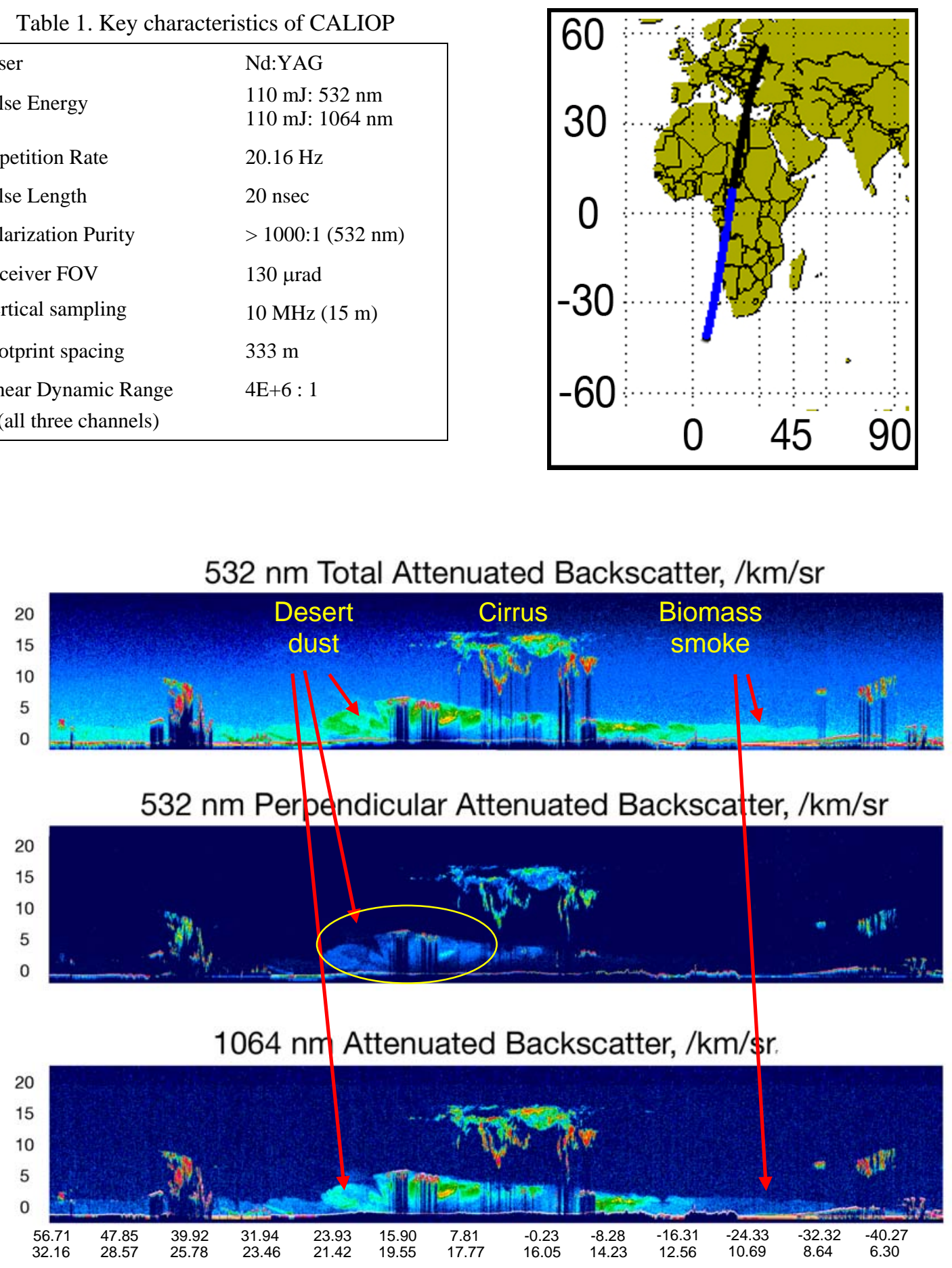

Figure 1. CALIOP observations from 9 June 2006, during the first week of on-orbit operation. The three panels show Level 1 data acquired along the orbit track shown in the map inset, from northern Europe across Africa into the south Atlantic. In the color coding scheme used in these panels, red represents strong returns from clouds and from the surface. Greens and yellows represent weak cloud and strong aerosol scattering, blues represent weak aerosol and molecular scattering. The middle panel indicates that depolarizing targets (cirrus and dust) produce a perpendicular return signal which is typically $1 / 4$ to $1 / 2$ of the parallel return signal. 


\section{On-orbit performance and initial observations}

CALIOP 'first light' occurred on 7 June 2006. As this is written, CALIOP has completed 5 months of nearcontinuous operation and initial assessments indicate excellent on-orbit performance. Energy-normalized return signals are about $30 \%$ greater than predicted by the instrument performance model, probably due to overly conservative estimates of optical transmission losses in the instrument. Laser pulse energy has been quite stable over this period, decreasing about 3\%, which is within expectations and has negligible impact on data quality. If necessary, pulse energy can be increased by increasing the length of the laser pump pulses.

Figure 1 shows an example of Level 1 data from the three lidar channels: $532 \mathrm{~nm}$ total and perpendicular attenuated backscatter, and $1064 \mathrm{~nm}$ total attenuated backscatter ("attenuated backscatter" is calibrated return signal, not yet corrected for attenuation). The image shows a nighttime transect from northern Europe southward across Africa to the southern Atlantic Ocean. Inspection of these three images illustrates the capability of CALIPSO to observe aerosol underneath cirrus and to discriminate different aerosol types.

High cirrus is seen in the center of the image, located over tropical Africa. The backscatter signal from the cirrus is similar at both wavelengths, characteristic of the relatively large cirrus particles. The cirrus is strongly depolarizing and produces a significant signal in the perpendicular channel. To the south of the cirrus (on the right) is a smoke layer originating from biomass fires in southern Africa, which are widespread during this time of year. Unlike the cirrus, this aerosol is weakly scattering at $1064 \mathrm{~nm}$, non-depolarizing, and produces negligible signal in the perpendicular channel.

The aerosol immediately to the north of the cirrus (toward the left) is primarily dust. Dust particles are relatively large and irregular, so they also produce strong signals in the $1064 \mathrm{~nm}$ and perpendicular channels. It can be seen that the aerosol north of about $25^{\circ} \mathrm{N}$ is non-depolarizing and more weakly scattering at $1064 \mathrm{~nm}$. This signature is indicative of secondary aerosol originating from anthropogenic activity.

CALIOP is the first satellite lidar to be optimized for observing aerosols and clouds. The new remote sensing capabilities provided by CALIOP will allow fundamental advances in our understanding of aerosols and clouds, and their role in the climate system.

\section{References}

[1] Winker, D. M., J. Pelon, and M. P. McCormick, 2003: The CALIPSO mission: Spaceborne lidar for observation of aerosols and clouds. Proc. SPIE 4893, 1-11.

[2] Winker, D. M., W. H. Hunt, and C. A. Hostetler, 2004: “Status and Performance of the CALIOP lidar,” Proc. SPIE, 5575, 8-15.

[3] Vaughan, M. A., S. A. Young, D. M. Winker, K. A. Powell, A. H. Omar, Z. Liu, Y. Hu, and C. A. Hostetler, 2004: "Fully automated analysis of space-based lidar data: An overview of the CALIPSO retrieval algorithms and data products,” Proc. SPIE 5575, 16-30. 